\title{
Resonant Characteristics in Sandwich Gratings
}

\author{
Xuehui Xiong \\ School of Chemical and Environmental Engineering, Jianghan University, Wuhan, China \\ Email: xuehuixiong@163.com
}

How to cite this paper: Xiong, X.H. (2020) Resonant Characteristics in Sandwich Gratings. Journal of Applied Mathematics and Physics, 8, 3070-3074. https://doi.org/10.4236/jamp.2020.812225

Received: December 10, 2020 Accepted: December 26, 2020 Published: December 29, 2020

Copyright $\odot 2020$ by author(s) and Scientific Research Publishing Inc. This work is licensed under the Creative Commons Attribution International License (CC BY 4.0).

http://creativecommons.org/licenses/by/4.0/

\begin{abstract}
In this paper, the intensity distributions of the magnetic field in the proposed sandwich grating were studied. The results indicated that there were two apparent enhanced transmission peaks. The magnetic intensity distributions of these two peaks manifest that the narrow higher transmission enhancement peak was caused by guided mode resonance and the other wide low one was formed by surface plasmon resonance. The resonant wavelength was estimated by the momentum matching conditions of resonance.
\end{abstract}

\section{Keywords}

Sandwich Grating, Guided Mode Resonance, Surface Plasmon Resonance

\section{Introduction}

Subwavelength structures on the surface of a metal film can strongly modify its interaction with electromagnetic fields [1]. Furthermore, by placing two such metallic layers in close proximity, the strong interaction between the evanescent fields on the surfaces of two or more nanostructured metal layers could lead to novel optical properties and offer new functionalities. Many double-layer metallic subwavelength structures have been proposed such as double-layer, laterally shifted metallic subwavelength hole arrays [2], double-layer metallic subwavelength slit arrays [1], double-layer close-packed metallic gratings [3] and double-layer stacking metallic gratings with subwavelength slits [4]. These structures have been demonstrated to exhibit extraordinarily high transmission. In fact the planar dielectric gratings on thin metallic films without slits or holes can also increase the transmitted efficiency and acquire even higher transmission enhancement [5] [6] [7]. We have studied a kind of sandwich grating (SG) in which the silver thin film is sandwiched by two identical planar dielectric gratings [8]. In this paper, we emphasize on intensity distributions of the near field in the proposed sandwich grating (SG). The numerical calculations based on the expanded Rigorous 
Coupled-Wave Analysis (RCWA) [8] for the intensity distributions of the magnetic field $\left(|\mathrm{Hy}|^{2}\right)$ help us distinct the enhanced mechanism. And we estimate the resonance wavelengths according to the momentum matching conditions of resonance.

\section{The Sandwich Grating}

Figure 1 depicts the basic configuration of the proposed sandwich structure gratings. Thin silver film of thickness $h$ is sandwiched into two identical planar sinusoidal dielectric gratings. The lossless planar dielectric grating [9] [10] is characterized by a periodical medium. The relative permittivity can be depicted by

$$
\varepsilon_{2}(x, z)=\varepsilon_{4}(x, z)=\varepsilon_{a v g}+\Delta \varepsilon \cos [K(x \sin \phi+z \cos \phi)],
$$

where $\varepsilon_{\text {avg }}$ is the average permittivity and $\Delta \varepsilon$ is the amplitude of the sinusoidal permittivity. $\phi$ is the grating slant angle and $K=2 \pi / \Lambda$, here $\Lambda$ is the grating period.

The Drude model is adopted to simulate the sandwiched Ag film in the region III with plasmon frequency $\omega_{p}=1.37 \times 10^{16} \mathrm{rad} / \mathrm{s}$ and the collision frequency $\gamma=7.29 \times 10^{13} \mathrm{rad} / \mathrm{s}$ [11]. The surrounding permittivity in the region I and $\mathrm{V}$ is chosen to be $1.33^{2}$. The detailed structure parameters are listed below: the grating period $\Lambda=400 \mathrm{~nm}$, the grating thickness $d=100 \mathrm{~nm}$, the average permittivity is 2.25 and the modulation is 0.33 . The sandwiched silver thin film thickness is set to be $40 \mathrm{~nm}$. The normal incidence wavelengths we are interested in the visible light range from $390 \mathrm{~nm}$ to $760 \mathrm{~nm}$.

\section{Numerical Calculations and Discussions}

According to the expanded rigorous coupled-wave analysis theory (RCWA) for the sandwich grating [8] [9] [10], we calculate the diffractive efficiencies of

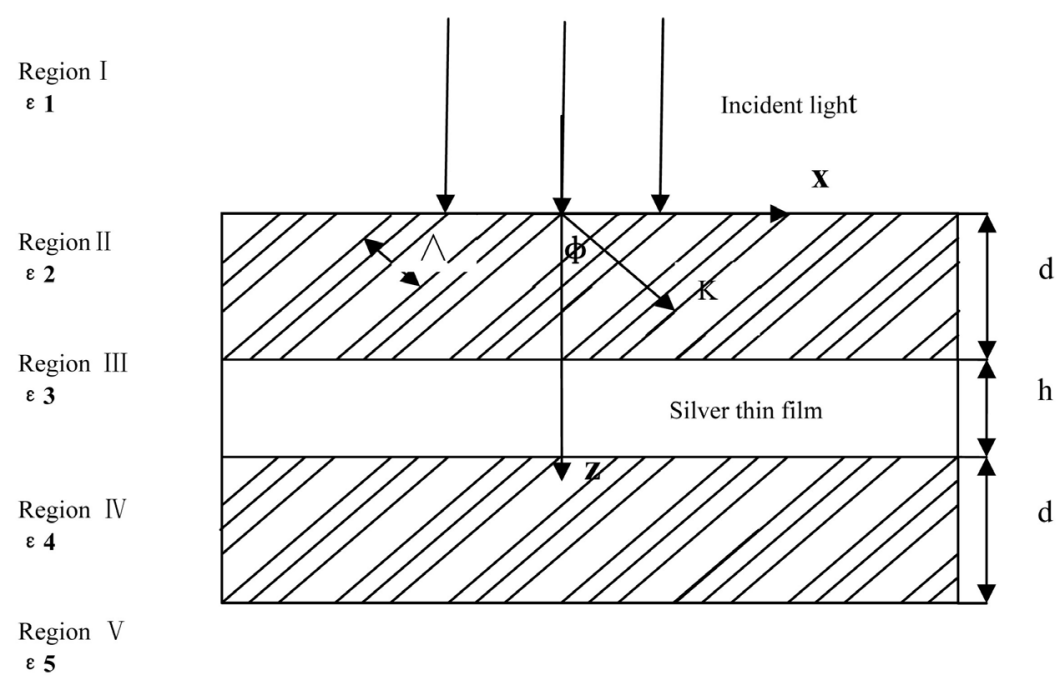

Figure 1. Schematic diagram of the sandwich grating. 
E-mode polarization in sandwich structures. For E-mode polarization, the magnetic field is solely in the y-direction. The computed 0th order transmission spectrum is shown in Figure 2, where two resonant peaks are observed. One narrow peak is at the short-wavelength of $596 \mathrm{~nm}$ whose transmission reaches 0.613364 . The other wider peak is at $647 \mathrm{~nm}$, whose transmission is 0.337835 .

Figure 3 visualizes the resonance mode profiles with $\lambda=596 \mathrm{~nm}$. The red and blue colors denote the maximum and minimum of magnetic intensity, respectively. At first, both interfaces between metallic films and gratings bound enhanced energy than other regions, which demonstrates that there forms resonant surface waves either guided or SPP modes [7]. Secondly, the resonant modes are the standing Bloch waves along $\mathrm{x}$-axis within a grating period $(\Lambda)$ due to the regenerating two opposite direction propagating resonant modes at normal incidence. Thirdly, high intensity in the thin metallic film layer (100 nm - $140 \mathrm{~nm}$ in the direction of $\mathrm{z}$ ) indicate that metallic film act as a light passageway. As a consequence, the absorption is only Ohm losses. So we can infer that the narrow resonant peak is corresponding to the guided mode resonance which cannot absorb extra energy. We estimate the resonance frequencies whether meeting a momentum matching condition. If the wave vector of guided or SPP modes matches that of scattered light, namely,

$$
k_{\mathrm{spp} / \text { guided }}=k_{x}+m K
$$

guided or SPP modes can be excited, where $K=2 \pi / \Lambda$ is the primitive reciprocal lattice vector and $\mathrm{m}$ is an integer [4]. According to this matching condition, the predicted wavelength of guided mode equals the average index of grating times grating period. The under-estimated wavelength is $600 \mathrm{~nm}$, very close to the resonant wavelength $596 \mathrm{~nm}$.

On the contrary, in Figure 4, the energy in the metallic film is very few, which manifests that the energy is strongly absorbed by the surface plasmon resonance besides ohm losses of guided resonance. According to this matching condition $\lambda_{\mathrm{spp}}=\Lambda n_{\mathrm{spp}}=634 \mathrm{~nm}$, close to the resonant wavelength $647 \mathrm{~nm}$.

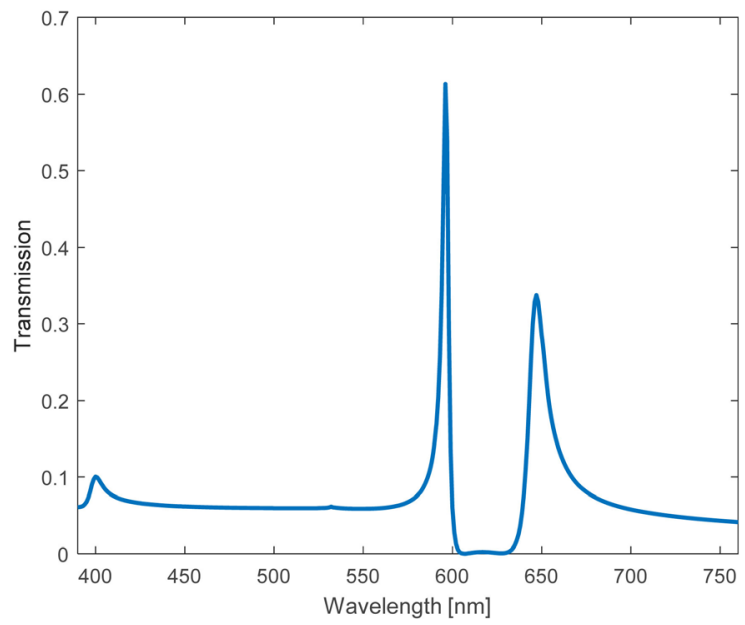

Figure 2. The computed 0th order transmission spectrum. 

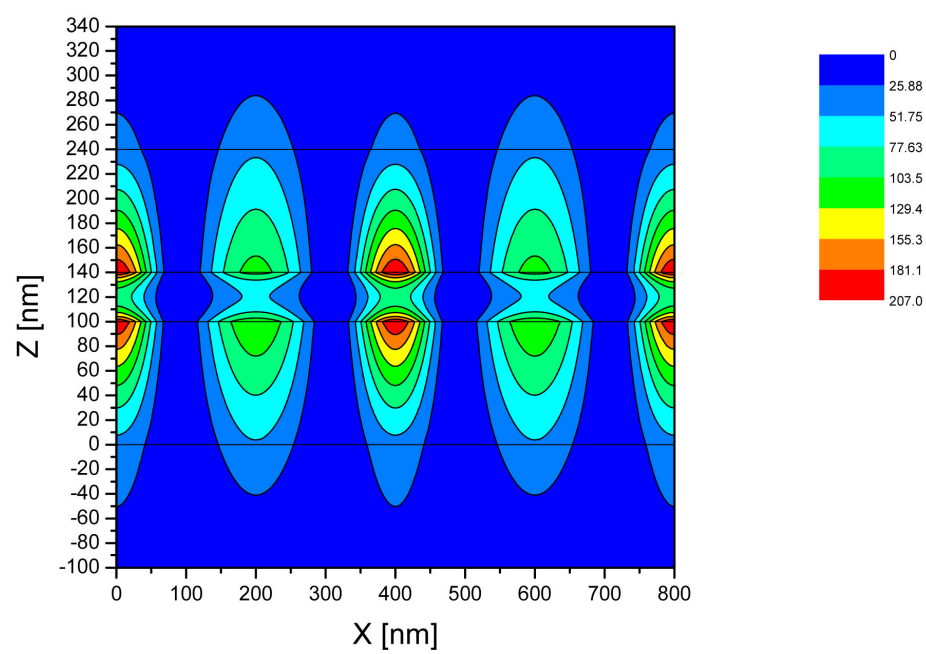

Figure 3. The contour profiles of intensity distributions of the magnetic field $\left(\left|\mathrm{H}_{\mathrm{y}}\right|^{2}\right)$ at the transmission peak wavelength of $596 \mathrm{~nm}$.

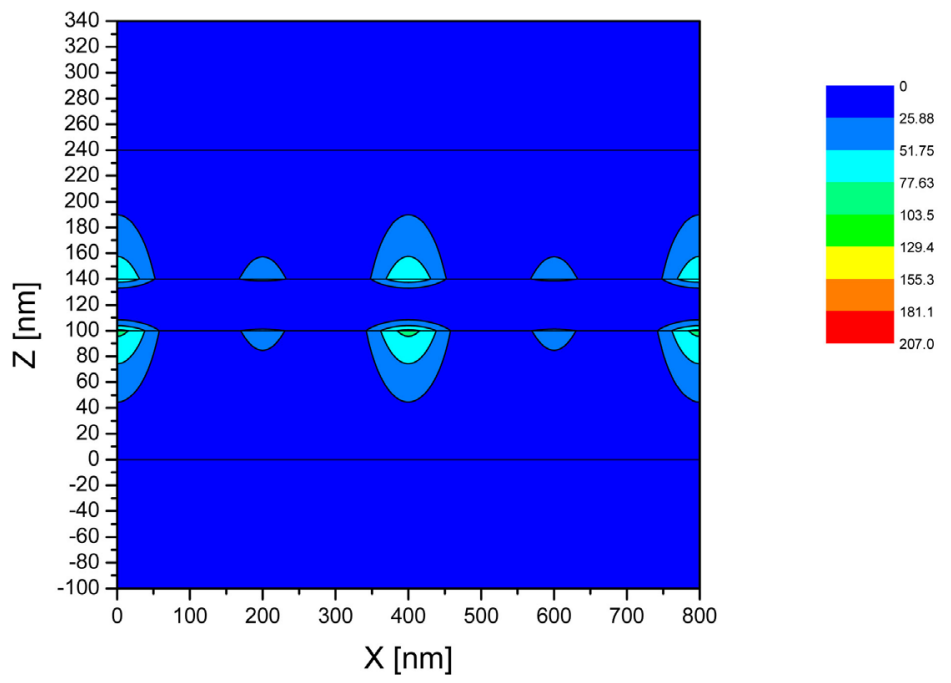

Figure 4. The contour profiles of intensity distributions of the magnetic field $\left(\left|\mathrm{H}_{\mathrm{y}}\right|^{2}\right)$ at the transmission peak wavelength of $647 \mathrm{~nm}$.

\section{Conclusion}

The results indicate that there are two apparent enhanced transmission peaks. The magnetic intensity distributions of these two peaks manifest that the narrow higher transmission enhancement peak is caused by guided mode resonance and the other wide low one is formed by surface plasmon resonance. The resonant wavelength was estimated by the momentum matching conditions of resonance. The completely planar structure would be exploited in the applications of nano-photonics circuits and surface plasmon resonance sensors.

\section{Conflicts of Interest}

The author declares no conflicts of interest regarding the publication of this paper. 


\section{References}

[1] Chan, H.B., Marcet, Z., Woo, K., Tanner, D.B., and Carr, D.W. (2006) Optical Transmission through Double-Layer Metallic Subwavelength Slit Arrays. Optics letters, 31, 516-518. https://doi.org/10.1364/OL.31.000516

[2] Marcet, Z., Hang, Z.H., Chan, C.T., Kravchenko, I., Bower, J.E., Cirelli, R.A., Klemens, F., Mansfield, W.M., Miner, J.F., Pai, C.S. and Chan, H.B. (2010) Optical Transmission through Double-Layer, Laterally Shifted Metallic Subwavelength Hole Arrays. Optics Letters, 35, 2124-2126. https://doi.org/10.1364/OL.35.002124

[3] Zhou, L., Wang, Q., Wu, S., Huang, W., Huang, C. and Zhu, Y. (2011) Hybrid of Surface Plasmon Polaritons and Waveguide Resonances through Double-Layer Metallic Gratings. Journal of the Optical Society of America B, 28,587-591. https://doi.org/10.1364/JOSAB.28.000587

[4] Fong, K. and Huia, P.M. (2006) Coupling of Waveguide and Surface Modes in Enhanced Transmission through Stacking Gratings. Applied Physics Letters, 89, Article ID: 091101-3. https://doi.org/10.1063/1.2338796

[5] Park, S., Lee, G., Song, S.H., Oh, C.H. and Kim, R.S. (2003) Resonant Coupling of Surface Plasmons to Radiation Modes by Use of Dielectric Gratings. Optics Letters, 28, 1870-1872. https://doi.org/10.1364/OL.28.001870

[6] Byun, K.M., Kim, S.J. and Kim, D. (2007) Grating-Coupled Transmission-Type Surface Plasmon Resonance Sensors Based on Dielectric and Metallic Gratings. Applied Optics, 46, 5703-5708. https://doi.org/10.1364/AO.46.005703

[7] Lenaerts, C., Michel, F., Tilkens, B., Lion, Y. and Renotte, Y. (2005) Highly Transmission Efficiency for Surface Plasmon Resonance by Use of a Dielectric Grating. Applied Optics, 44, 6017-6022. https://doi.org/10.1364/AO.44.006017

[8] Xiong, X. and Lu, P. (2016) Rigorous Coupled-Wave Approach for Sandwich Gratings. Journal of Applied Mathematics and Physics, 4, 1439-1445.

https://doi.org/10.4236/jamp.2016.48148

[9] Moharam, M.G. and Gaylord, T.K. (1981) Rigorous Coupled-Wave Analysis of Planar-Grating Diffraction. Journal of the Optical Society of America, 71, 811-818. https://doi.org/10.1364/JOSA.71.000811

[10] Moharam, M.G. and Gaylord, T.K. (1983) Rigorous Coupled-Wave Analysis of Grating Diffraction-E-Mode Polarization and Losses. Journal of the Optical Society of America, 73, 451-455. https://doi.org/10.1364/JOSA.73.000451

[11] Rakić, A.D., Djurišić, A.B., Elazar, J.M. and Majewski. M.L. (1998) Optical Properties of Metallic Films for Vertical-Cavity Optoelectronic Devices. Applied Optics, 37, 5271-5283. https://doi.org/10.1364/AO.37.005271 